\title{
Physical Properties of Sixty API-NBS Hydrocarbons
}

\author{
By Alphonse F. Forziati ${ }^{2}$ and Frederick D. Rossini
}

\begin{abstract}
The results of experimental determinations of the density at $20^{\circ}, 25^{\circ}$, and $30^{\circ} \mathrm{C}$, the refractive index, $n_{D}$, at $20^{\circ}, 25^{\circ}$, and $30^{\circ} \mathrm{C}$, and the boiling point and pressure coefficient of the boiling point, at 1 atmosphere, are reported for 60 hydrocarbons of the API-NBS series, including 17 paraffins, 14 alkylcyclopentanes, 8 alkylcyclohexanes, and 21 alkylbenzenes.
\end{abstract}

\section{Introduction}

As part of the work of the American Petroleum Institute Research Project 6 on the fractionation, analysis, purification, and properties of hydrocarbons, measurements are being made of the physical properties of the API-NBS series of highly purified hydrocarbons. This paper gives the results of measurements of refractive index, density, and boiling point of 60 API-NBS hydrocarbons, including 17 paraffins, 14 alkylcyclopentanes, 8 alkylcyclohexanes, and 21 alkylbenzenes.

\section{Compounds Investigated}

The compounds whose properties were measured in the present investigation were samples from the API-NBS series of highly purified hydrocarbons, which are being prepared through a cooperative undertaking of the American Petroleum Institute and the National Bureau of Standards.

These samples of API-NBS hydrocarbons have been made available by the American Petroleum Institute and the National Bureau of Standards through the API Research Project 44 on the "Collection, Analysis, Calculation, and Compilation of Data on the Properties of Hydrocarbons". The samples were purified at the National Bureau of Standards by the API Research Project 6 on the "Analysis, Purification, and Properties of Hydrocarbons", from material supplied by the following laboratories:

1 This investigation was performed at the National Bureau of Standards as part of the work of the American Petroleum Institute Research Project 6 on the "Analysis, Purification, and Properties of Hydrocarbons."

2 Research Associate on the American Petroleum Institute Research Project 6 at the National Bureau of Standards.
$n$-Heptane, 2,4-dimethylpentane, n-nonane, 2,2,5-trimethylhexane, benzene, 1,2,3-trimethylbenzene, and 1,2,4-trimethylbenzene, by the American Petroleum Institute Research Project 6 at the National Bureau of Standards.

2-Methylhexane and 3-methylhexane, by the Ethyl Corporation, Detroit, Mich.

3-Ethylpentane, 3,3-dimethylpentane, 3,3-diethylpentane, 2,2,3,3-tetramethylpentane, cis-1,3dimethylcyclopentane, trans-1,3-dimethylcyclopentane, $n$-propylcyclopentane, isopropylcyclopentane, 1,1-dimethylcyclohexane, $n$-propylcyclohexane, isopropylcyclohexane, 1,1,3-trimethylcyclohexane, n-butylcyclohexane, isobutylcyclohexane, sec-butylcyclohexane, tert-butylcyclohexane, 1,3-dimethylbenzene, 1,4-dimethylbenzene, 1-methyl-2-ethylbenzene, 1-methyl-3-ethylbenzene, 1,3,5-trimethylbenzene, $n$-butylbenzene, isobutylbenzene, sec-butylbenzene, and tert-butylbenzene, by the American Petroleum Institute Research Project 45, at the Ohio State University, Columbus, Ohio, under the supervision of C. E. Boord.

2,2-Dimethylpentane, by the Socony-Vacuum Laboratories, Paulsboro, N. J.

2,3-Dimethylpentane and 2,2,3-trimethylbutane, by General Motors Corporation, Detroit, Mich.

2,4,4-Trimethylhexane, 1,1-dimethylcyclopentane, cis-1,2-dimethylcyclopentane, trans-1,2dimethylcyclopentane, 1,1,2-trimethylcyclopentane, cis,cis,trans-1,2,4-trimethylcyclopentane, cis,trans,cis-1,2,4-trimethylcyclopentane, 1-methyl-1-ethylcyclopentane, and 1-methyl-cis-2-ethylcyclopentane, by the Hydrocarbon Laboratory, Pennsylvania State College, State College, Pa. 
2,2,3,4-Tetramethylpentane, 2,2,4,4-tetramethylpentane, 2,3,3,4-tetramethylpentane, 1-methyl4-ethylbenzene, 1,2-diethylbenzene, and 1,4diethylbenzene, by the National Advisory Committee for Aeronautics, Flight Propulsion Research Laboratory, Cleveland, Ohio.

1,1,3-Trimethylcyclopentane, by the American Petroleum Institute Research Project 45, at the Ohio State University, Columbus, Ohio, and the Gulf Oil Company Fellowship, at the Mellon Institute of Industrial Research, Pittsburgh, Pa.

Ethylcyclopentane, by the American Petroleum Institute Research Project 45, Ohio State University, Columbus, Ohio, and the Hydrocarbon Laboratory, Pennsylvania State College, State College, Pa.

Methylbenzene, by the Humble Oil and Refining Company, Houston, Tex.

Ethylbenzene and isopropylbenzene, by the Monsanto Chemical Company, Dayton, Ohio.

1,2-Dimethylbenzene, by the Standard Oil Development Company, Elizabeth, N. J.

$n$-Propylbenzene, by the Dow Chemical Company, Midland, Mich.

1,3-Diethylbenzene, by the National Advisory Committee for Aeronautics, Flight Propulsion Research Laboratory, Cleveland, Ohio, and the American Petroleum Institute Research Project 45 at the Ohio State University, Columbus, Ohio.

The purification and determination of purity and freezing points of these compounds are described in references $[1,2,3,4,5] .^{3}$

\footnotetext{
${ }^{3}$ Figures in brackets indicate the literature references at the end of this paper.
}

It is believed that in each case the impurity was of such nature and present in such small amount that the properties measured were not affected beyond the indicated limits of uncertainty.

\section{Refractive Indices}

The measurements of refractive index were made with a precision refractometer, ${ }^{4}$ by reference to the three hydrocarbons, 2,2,4-trimethylpentane, methylcyclohexane, and methylbenzene (toluene), for which the values of refractive index were determined on a spectrometer with hollow prism by L. W. Tilton, of the Optical Instruments Section of the National Bureau of Standards. These three hydrocarbons are NBS Standard Samples of hydrocarbons certified with respect to refractive index at $20^{\circ}, 25^{\circ}$, and $30^{\circ} \mathrm{C}$. The values of refractive index for the three reference hydrocarbons were reported with an accuracy of \pm 0.00001 , and were used as follows: 2,2,4-trimethylpentane for the paraffin hydrocarbons, methylcyclohexane for the cycloparaffin hydrocarbons, and methylbenzene for the alkylbenzene hydrocarbons. By this procedure, the refractometer was actually used only to determine the difference in refractive index, $n_{D}$, between the reference compound and the hydrocarbon under investigation. The experimental results on refractive index are given in table 1 . Individual measurements were reproducible within \pm 0.00002 to \pm 0.00003 . The accuracy of the tabulated values, including the effect of impurities, is estimated to be \pm 0.00005 to \pm 0.00008 .

\footnotetext{
${ }^{4}$ Modified Abbe-type, Bausch and Lomb.
} 
TABLE 1. Values of refractive index, density, and boiling point for sixty API-NBS hydrocarbons

\begin{tabular}{|c|c|c|c|c|c|c|c|c|c|c|c|}
\hline \multirow{2}{*}{ Compound } & \multirow{2}{*}{ Formula } & \multirow{2}{*}{$\begin{array}{c}\text { Boiling } \\
\text { point at } \\
760 \mathrm{~mm} \\
\mathrm{Hg}\end{array}$} & \multirow{2}{*}{$\begin{array}{c}\text { Pressure } \\
\text { coefficient } \\
\text { of boiling } \\
\text { point at } 760 \\
\text { mm Hg } \\
d t / d P\end{array}$} & \multicolumn{3}{|c|}{ Refractive index a $n_{\mathrm{D}}$} & \multirow{2}{*}{$\begin{array}{l}\text { Tempera- } \\
\text { ture coeffi- } \\
\text { cient of re- } \\
\text { fractive in- } \\
\text { dex }\left(n_{\mathrm{D}}\right) \\
\text { at } 25^{\circ} \mathrm{C}\end{array}$} & \multicolumn{3}{|c|}{ Density a } & \multirow{2}{*}{$\begin{array}{l}\text { Tempera- } \\
\text { ture coeffi- } \\
\text { cient of } \\
\text { density at } \\
25^{\circ} \mathrm{C}\end{array}$} \\
\hline & & & & $20^{\circ} \mathrm{C}$ & $25^{\circ} \mathrm{C}$ & $30^{\circ} \mathrm{C}$ & & $20^{\circ} \mathrm{C}$ & $25^{\circ} \mathrm{C}$ & $30^{\circ} \mathrm{C}$ & \\
\hline \multicolumn{12}{|c|}{ PARAFFINS } \\
\hline$n$-Heptan & $\mathrm{C}_{7} \mathrm{H}_{16}$ & $\begin{array}{l}{ }^{\circ} C \\
98.427\end{array}$ & $\begin{array}{r}{ }^{\circ} \mathrm{C} / \mathrm{mmHg} \\
0.04481\end{array}$ & 1. 38764 & 1. 38511 & 1. 38258 & $\begin{array}{c}1 /{ }^{\circ} C \\
0.000506\end{array}$ & $\begin{array}{c}g / m l \\
0.68376\end{array}$ & $\begin{array}{c}g / m l \\
0.67951\end{array}$ & $\begin{array}{c}g / m l \\
0.67526\end{array}$ & $\begin{array}{l}g / m l{ }^{\circ} \mathrm{C} \\
0.000850\end{array}$ \\
\hline 2-Methylhexane & $\mathrm{C}_{7} \mathrm{H}_{16}$ & 90.052 & $\begin{array}{r}0.07401 \\
.04431\end{array}$ & 1. 38485 & 1. 38227 & $\begin{array}{l}\text { 1. } 08258 \\
1.37969\end{array}$ & $\begin{array}{r}.000000 \\
.000516\end{array}$ & $\begin{array}{r}0.08070 \\
.67859\end{array}$ & $\begin{array}{r}0.07951 \\
.67439\end{array}$ & $\begin{array}{r}0.06020 \\
.67001\end{array}$ & $\begin{array}{r}0.000800 \\
.000858\end{array}$ \\
\hline 3-Methylhexane ... & $\mathrm{C}_{7} \mathrm{H}_{16}$ & 91.850 & .04459 & 1. 38864 & 1. 38609 & 1. 38354 & .000510 & .68713 & .68295 & .67852 & .000861 \\
\hline 3-Ethylpentane $\ldots . . .$. & $\mathrm{C}_{7} \mathrm{H}_{16}$ & 93.475 & .04482 & 1. 39339 & 1. 39084 & 1. 38829 & .000510 & .69816 & .69395 & .68948 & .000868 \\
\hline 2,2-Dimethylpentane .... & $\mathrm{C}_{7} \mathrm{H}_{16}$ & 79.197 & .04394 & 1. 38215 & 1. 37955 & 1. 37695 & .000520 & .67385 & .66953 & .66508 & .000877 \\
\hline 2,3-Dimethylpentane .... & $\mathrm{C}_{7} \mathrm{H}_{16}$ & 89. 784 & .04482 & 1. 39196 & 1. 38945 & 1. 38694 & .000502 & .69508 & .69091 & .68650 & .000858 \\
\hline 2,4-Dimethylpentane $\ldots . . . . . . . .$. & $\mathrm{C}_{7} \mathrm{H}_{16}$ & 80.500 & .04376 & 1. 38145 & 1. 37882 & 1. 37619 & .000526 & .67270 & .66832 & .66383 & .000887 \\
\hline 3,3-Dimethylpentane $\ldots \ldots . .$. & $\mathrm{C}_{7} \mathrm{H}_{16}$ & 86. 064 & .04509 & 1. 39092 & 1. 38842 & 1. 38592 & .000500 & .69327 & .68908 & .68481 & .000846 \\
\hline 2,2,3-Trimethylbutane.... & $\mathrm{C}_{7} \mathrm{H}_{16}$ & 80.882 & .04484 & 1. 38944 & 1. 38692 & 1. 38440 & .000504 & .69011 & 68588 & .68154 & .000857 \\
\hline$n$-Nonane $\ldots$ & $\mathrm{C}_{9} \mathrm{H}_{20}$ & 150. 798 & .04967 & 1. 40542 & 1. 40311 & 1. 40080 & .000462 & .71763 & .71381 & .70994 & .000768 \\
\hline 2,2,5-Trimethylhexane ... & $\mathrm{C}_{9} \mathrm{H}_{20}$ & 124.084 & .04838 & 1. 39972 & 1. 39728 & 1. 39484 & .000488 & .70721 & .70322 & .69905 & .000816 \\
\hline 2,4,4-Trimethylhexane & $\mathrm{C}_{9} \mathrm{H}_{20}$ & 130.648 & .04960 & 1. 40745 & 1. 40515 & 1.40285 & .000460 & .72381 & .72007 & .71612 & .000769 \\
\hline 3,3-Diethylpentane ................. & $\mathrm{C}_{9} \mathrm{H}_{20}$ & 146. 168 & .05109 & 1. 42051 & 1. 41837 & 1. 41623 & .000428 & .75359 & .75000 & .74634 & .000725 \\
\hline 2,2,3,3-Tetramethylpentane ........ & $\mathrm{C}_{9} \mathrm{H}_{20}$ & 140. 274 & .05124 & 1. 42360 & 1. 42140 & 1. 41920 & .000440 & .75666 & .75299 & .74925 & .000741 \\
\hline $2,2,3,4$-Tetramethylpentane $\ldots . . . . .$. & $\mathrm{C}_{\ominus} \mathrm{H}_{20}$ & 133.016 & .05030 & 1. 41472 & 1. 41246 & 1. 41020 & .000452 & .73895 & .73524 & .73144 & .000751 \\
\hline 2,2,4,4-Tetramethylpentane $\ldots . . . . .$. & $\mathrm{C}_{0} \mathrm{H}_{20}$ & 122. 284 & .04937 & 1. 40694 & 1. 40459 & 1. 40224 & .000470 & .71947 & .71563 & .71167 & .000780 \\
\hline 2,3,3,4-Tetramethylpentane........ & $\mathrm{C}_{2} \mathrm{H}_{20}$ & 141.551 & .04223 & 1. 42222 & 1. 42003 & 1. 41784 & .000438 & .75473 & .75113 & .74740 & .000733 \\
\hline
\end{tabular}

ALKYLCYCLOPENTANES

Ethylcylopentane

1,1-Dimethycyclopentane...

cis-1,2-Dimethylcyclopentane

trans-1,2-Dimethylcylopentane

cis-1,3-Dimethylcyclopentane.

trans-1,s-Dimethylcyclopentane

$n$-Propylcyclopentane

Isopropycyclopentane

1-Methyl-1-ethylcyclopentane

1-Methyl-cis-2-ethycyclopentane

1-1-2, Trimethylcyclopentane.

1,1,3-Trimethylcyclopentane...

cis, cis, trans-1,2,4-Trimethylcyclo-

pentane.

cis, trans, cis-1,2,4-Trimethylcyclo-

pentane.
$\mathrm{C}_{7} \mathrm{H}_{16}$

$\mathrm{C}_{7} \mathrm{H}_{1}$

$\mathrm{C}_{9} \mathrm{H}_{20}$

$\mathrm{C}_{9} \mathrm{H}_{20}$

$\mathrm{C}_{2} \mathrm{H}_{20}$

\begin{tabular}{l|r|r|r|r|r|r|r|r|r|r}
$\mathrm{C}_{7} \mathrm{H}_{14}$ & 103.466 & 0.04623 & 1.41981 & 1.41730 & 1.41479 & 0.000502 & 0.76647 & 0.76217 & 0.75780 & 0.000867 \\
$\mathrm{C}_{7} \mathrm{H}_{14}$ & 87.846 & .04497 & 1.41356 & 1.41091 & 1.40826 & .000530 & .75448 & .74991 & .74532 & .000916 \\
$\mathrm{C}_{7} \mathrm{H}_{14}$ & 99.532 & .04603 & 1.42217 & 1.41963 & 1.41709 & .000508 & .77262 & .76807 & .76364 & .000898 \\
$\mathrm{C}_{7} \mathrm{H}_{14}$ & 91.869 & .04521 & 1.41200 & 1.40941 & 1.40682 & .000518 & .75144 & .74686 & .74241 & .000903 \\
$\mathrm{C}_{7} \mathrm{H}_{14}$ & 91.725 & .04525 & 1.41074 & 1.40813 & 1.40552 & .000522 & .74880 & .74435 & .73985 & .000895 \\
$\mathrm{C}_{7} \mathrm{H}_{14}$ & 90.773 & .04518 & 1.40894 & 1.40633 & 1,40372 & .000522 & .74479 & .74025 & .73573 & .000906 \\
$\mathrm{C}_{8} \mathrm{H}_{16}$ & 130.949 & .04888 & 1.42626 & 1.42389 & 1.42152 & .000474 & .77633 & .77229 & .76811 & .000822 \\
$\mathrm{C}_{8} \mathrm{H}_{16}$ & 126.419 & .04913 & 1.42582 & 1.42350 & 1.42118 & .000464 & .77653 & .77259 & .76851 & .000802 \\
$\mathrm{C}_{8} \mathrm{H}_{16}$ & 121.522 & .04863 & 1.42718 & 1.42476 & 1.42234 & .000484 & .78093 & .77670 & .77260 & .000833 \\
$\mathrm{C}_{8} \mathrm{H}_{16}$ & 128.050 & .04897 & 1.42933 & 1.42695 & 1.42457 & .000476 & .78522 & .78113 & .77698 & .000824 \\
$\mathrm{C}_{8} \mathrm{H}_{16}$ & 113.729 & .04818 & 1.42298 & 1.42051 & 1.41804 & .000494 & .77252 & .76817 & .76399 & .000853 \\
$\mathrm{C}_{8} \mathrm{H}_{16}$ & 104.893 & .04724 & 1.41119 & 1.40870 & 1.40621 & .000498 & .74825 & .74392 & .73958 & .000867 \\
& & & & & & & & .752 & .759 \\
$\mathrm{C}_{8} \mathrm{H}_{16}$ & 116.731 & .04827 & 1.41855 & 1.41612 & 1.41369 & .000486 & .76345 & .75920 & .75504 & .000841 \\
$\mathrm{C}_{8} \mathrm{H}_{16}$ & 109.290 & .04738 & 1.41060 & 1.40812 & 1.40564 & .000496 & .74727 & .74302 & .73873 & .000854 \\
& & & & & & & &
\end{tabular}

ALKYLCYCLOHEXANES

1,1-Dimethylcyclohexane n-Propylcyclohexane

Isopropylcyclohexane

1,1,3-Trimethylcyclohexane

$n$-Butylcyclohexane

Isobutylcyclohexane.

sec-Butylcyclohexane-

tert-Butyleyclohexane.............

\begin{tabular}{l|l}
$\mathrm{C}_{8} \mathrm{H}_{16}$ & 119.543 \\
$\mathrm{C}_{9} \mathrm{H}_{18}$ & 156.724 \\
$\mathrm{C}_{9} \mathrm{H}_{18}$ & 154.563 \\
$\mathrm{C}_{9} \mathrm{H}_{18}$ & 136.626 \\
$\mathrm{C}_{10} \mathrm{H}_{20}$ & 180.947 \\
$\mathrm{C}_{10} \mathrm{H}_{20}$ & 171.321 \\
$\mathrm{C}_{10} \mathrm{H}_{20}$ & 179.335 \\
$\mathrm{C}_{10} \mathrm{H}_{20}$ & 171.591
\end{tabular}

\begin{tabular}{r|l|l|l}
0.04920 & 1.42900 & 1.42662 & 1.42424 \\
.05200 & 1.43705 & 1.43478 & 1.43251 \\
.05210 & 1.44087 & 1.43861 & 1.43635 \\
.05089 & 1.42955 & 1.42725 & 1.42495 \\
.05412 & 1.44075 & 1.43855 & 1.43635 \\
.05367 & 1.43861 & 1.43636 & 1.43411 \\
.05440 & 1.44673 & 1.44454 & 1.44235 \\
.05429 & 1.44694 & 1.44473 & 1.44252
\end{tabular}

\begin{tabular}{r|r|} 
& \\
0.000476 & 0.78094 \\
.000454 & .79360 \\
.000452 & .80221 \\
.000460 & .77883 \\
.000440 & .79918 \\
.000450 & .79521 \\
.000438 & .81314 \\
.000442 & .81267
\end{tabular}

0.77677
.78977
.79833
.77497
.79551
.79141
.80935
.80890

0.

ALKYLBENZENES

Benzene

Methylbenzene (Toluene)

Ethylbenzene ......................

1,2-Dimethylbenzene (o-Xylene) .-

1,3-Dimethylbenzene ( $m$-Xylene)

1,4-Dimethylbenzene ( $p$-Xylene)..

n-Propylbenzene

See footnote at end of table.

\begin{tabular}{l|r|r|r|r|r}
$\mathrm{C}_{6} \mathrm{H}_{6}$ & 80.099 & 0.04271 & 1.50112 & 1.49792 & 1.49478 \\
$\mathrm{C}_{7} \mathrm{H}_{8}$ & 110.626 & .04630 & 1.49693 & 1.49414 & 1.49126 \\
$\mathrm{C}_{8} \mathrm{H}_{10}$ & 136.186 & .04898 & 1.49588 & 1.49320 & 1.49050 \\
$\mathrm{C}_{8} \mathrm{H}_{10}$ & 144.411 & .04969 & 1.50545 & 1.50295 & 1.50032 \\
$\mathrm{C}_{8} \mathrm{H}_{10}$ & 139.104 & .04903 & 1.49722 & 1.49464 & 1.49198 \\
$\mathrm{C}_{8} \mathrm{H}_{10}$ & 138.351 & .04917 & 1.49582 & 1.49325 & 1.49054 \\
$\mathrm{C}_{9} \mathrm{H}_{12}$ & 159.218 & .05143 & 1.49202 & 1.48951 & 1.48702
\end{tabular}

\begin{tabular}{r|r|r|r|r}
0.000635 & 0.87901 & 0.87370 & 0.86846 & 0.001055 \\
.000567 & .86694 & .86230 & .85770 & .000924 \\
.000534 & .86702 & .86264 & .85828 & .000874 \\
.000513 & .88020 & .87596 & .87186 & .000834 \\
.000518 & .86417 & .85990 & .85565 & .000852 \\
.000537 & .86105 & .85669 & .85246 & .000859 \\
.000500 & .86204 & .85780 & .85370 & .000834
\end{tabular}


TABLE 1. Values of refractive index, density, and boiling point for sixty API-NBS hydrocarbons-Continued

\begin{tabular}{|c|c|c|c|c|c|c|c|c|c|c|c|}
\hline \multirow{2}{*}{ Compound } & \multirow{2}{*}{ Formlua } & \multirow{2}{*}{$\begin{array}{c}\text { Boiling } \\
\text { point at } \\
760 \mathrm{~mm} \\
\mathrm{Hg}\end{array}$} & \multirow{2}{*}{$\begin{array}{c}\text { Pressure } \\
\text { coefficient } \\
\text { of boiling } \\
\text { point at } 760 \\
\mathrm{~mm} \mathrm{Hg} \\
d t / d P\end{array}$} & \multicolumn{3}{|c|}{ Refractive index a $n_{\mathrm{D}}$} & \multirow{2}{*}{$\begin{array}{l}\text { Tempera- } \\
\text { ture coeffi- } \\
\text { cient of re- } \\
\text { fractive in- } \\
\text { dex }(n \mathrm{D}) \\
\text { at } 25^{\circ} \mathrm{C}\end{array}$} & \multicolumn{3}{|c|}{ Density a } & \multirow{2}{*}{$\begin{array}{l}\text { Tempera- } \\
\text { ture coeffi- } \\
\text { cient of } \\
\text { density at } \\
25^{\circ} \mathrm{C}\end{array}$} \\
\hline & & & & $20^{\circ} \mathrm{C}$ & $25^{\circ} \mathrm{C}$ & $30^{\circ} \mathrm{C}$ & & $20^{\circ} \mathrm{C}$ & $25^{\circ} \mathrm{C}$ & $30^{\circ} \mathrm{C}$ & \\
\hline \multicolumn{12}{|c|}{ ALKYLBENZENES-Continued } \\
\hline Isopropylbenzene (Cumene) & $\mathrm{C}_{9} \mathrm{H}_{12}$ & $\begin{array}{c}{ }^{\circ} C \\
152.392\end{array}$ & $\begin{array}{r}{ }^{\circ} \mathrm{C} / \mathrm{mmHg} \\
.05074\end{array}$ & 1. 49145 & 1. 48890 & 1. 48646 & $\begin{array}{l}1{ }^{\circ} \mathrm{C} \\
.000499\end{array}$ & $\begin{array}{l}g / m l \\
.86179\end{array}$ & $\begin{array}{l}g / m l \\
.85751\end{array}$ & $\begin{array}{l}g / m l \\
.85335\end{array}$ & $\begin{array}{r}g / m l \circ C \\
.000844\end{array}$ \\
\hline 1-Methyl-2-ethylbenzene .... & $\mathrm{C}_{9} \mathrm{H}_{12}$ & 165.153 & .05163 & 1. 50456 & 1.50208 & 1.49963 & .000493 & .88069 & .87657 & .87252 & .000817 \\
\hline 1-Methyl-3-ethylbenzene & $\mathrm{C}_{9} \mathrm{H}_{12}$ & 161.305 & .05111 & 1. 49660 & 1. 49406 & 1. 49154 & .000511 & .86452 & .86040 & .85630 & .000822 \\
\hline 1-Methyl-4-ethylbenzene $\ldots . . . . .$. & $\mathrm{C}_{9} \mathrm{H}_{12}$ & 161.989 & .05148 & 1. 49500 & 1. 49244 & 1. 48999 & .000497 & .86118 & .85702 & .85299 & .000819 \\
\hline $\begin{array}{l}\text { 1,2,3-Trimethylbenzene (Hemimel- } \\
\text { litene) }\end{array}$ & $\mathrm{C}_{9} \mathrm{H}_{12}$ & 176.084 & .05263 & 1. 51393 & 1. 51150 & 1. 50911 & .000482 & .89438 & .89044 & .88652 & .000786 \\
\hline $\begin{array}{l}\text { 1,2,4-Trimethylbenzene (Pseudo- } \\
\text { cumene) }\end{array}$ & $\mathrm{C}_{9} \mathrm{H}_{12}$ & 169.351 & .05187 & 1. 50484 & 1. 50237 & 1. 49990 & .000493 & .87582 & .87180 & .86782 & .000800 \\
\hline $\begin{array}{l}\text { 1,3,5-Trimethylbenzene (Mesity- } \\
\text { lene) }\end{array}$ & $\mathrm{C}_{9} \mathrm{H}_{12}$ & 164.716 & .05100 & 1. 49937 & 1. 49684 & 1. 49437 & .000500 & .86518 & .86111 & .85707 & .000811 \\
\hline$n$-Butylbenzene (l-Phenylbutane) & $\mathrm{C}_{10} \mathrm{H}_{14}$ & 183.270 & .05358 & 1. 48979 & 1. 48742 & 1. 48502 & .000477 & .86013 & .85607 & .85218 & .000795 \\
\hline $\begin{array}{l}\text { I s o b u t y l b e n z e n e } \\
\text { Phenyl-2-methylpropane) }\end{array}$ & $\mathrm{C}_{10} \mathrm{H}_{14}$ & 172. 759 & .05319 & 1. 48646 & 1. 48400 & 1. 48157 & .000492 & .85321 & .84907 & .84512 & .000809 \\
\hline $\begin{array}{l}\text { s e c-B u t y l be n } z \text { e n e } \quad(2- \\
\text { Phenylbutane }\end{array}$ & $\mathrm{C}_{10} \mathrm{H}_{14}$ & 173.305 & .05313 & 1. 49020 & 1. 48779 & 1. 48537 & .000482 & .86207 & .85797 & .85405 & .000802 \\
\hline $\begin{array}{l}\text { tert-B u t y lbenzene } \\
\text { Phenyl-2-methylpropane })\end{array}$ & $\mathrm{C}_{10} \mathrm{H}_{14}$ & 169.119 & .05269 & 1. 49266 & 1. 49024 & 1. 48776 & .000490 & .86650 & .86240 & .85834 & .000816 \\
\hline 1,2-Diethylbenzene & $\mathrm{C}_{10} \mathrm{H}_{14}$ & 183.423 & .05340 & 1. 50346 & 1. 50106 & 1. 49865 & .000481 & .87996 & .87592 & .87197 & .000799 \\
\hline 1,3-Diethylbenzene................. & $\mathrm{C}_{10} \mathrm{H}_{14}$ & 181.102 & .05293 & 1.49552 & 1. 49310 & 1. 49067 & .000485 & .86394 & .85993 & .85590 & .000804 \\
\hline 1,4-Diethylbenzene $\ldots \ldots$ & $\mathrm{C}_{10} \mathrm{H}_{14}$ & 183. 752 & .05351 & 1. 49483 & 1. 49245 & 1. 49003 & .000480 & .86196 & .85794 & .85402 & .000794 \\
\hline
\end{tabular}

a For air-saturated hydrocarbon in the liquid state at 1 atmosphere.

\section{Densities}

The measurements of density were made with a density balance, the assembly, calibration, and operation of which has been previously described [6]. The experimental results on density are given in table 1 . Individual measurements were reproducible within \pm 0.00002 to $\pm 0.00003 \mathrm{~g} / \mathrm{ml}$. The accuracy of the tabulated values, including the effect of impurities, is estimated to be \pm 0.00005 to $\pm 0.00010 \mathrm{~g} / \mathrm{ml}$.

\section{Boiling Points}

The normal boiling points of these compounds were determined as part of another investigation on their vapor pressure, and the values reported in table 1 for the normal boiling point and the pressure coefficient of the boiling point at 1 atmosphere are taken directly from the report of that investigation [7]. Individual measurements of the normal boiling point were reproducible within $\pm 0.002 \mathrm{deg}$ to $\pm 0.003 \mathrm{deg} \mathrm{C}$. The accuracy of the tabulated values of the normal boiling point, including the effect of impurities, is estimated to be $\pm 0.005 \mathrm{deg}$ to $\pm 0.010 \mathrm{deg} \mathrm{C}$.

\section{References}

[1] A. R. Glasgow, Jr., E. T. Murphy, C. B. Willingham, and F. D. Rossini, J. Research NBS 37, 141 (1946) RP1734.

[2] A. J. Streiff, E. T. Murphy, V. A. Sedlak, C. B. Willingham, and F. D. Rossini, J. Research NBS 37, 331 (1946) RP1752.

[3] A. J. Streiff, E. T. Murphy, J. C. Cahill, H. F. Flanagan, V. A. Sedlak, C. B. Willingham, and F. D. Rossini, J. Research NBS 38, 53 (1947) RP1760.

[4] A. J. Streiff, E. T. Murphy, J. C. Zimmerman, L. F. Soule, V. A. Sedlak, C. B. Willingham, and F. D. Rossini, J. Research NBS 39, 321 (1947) RP1833.

[5] A. J. Streiff, J. C. Zimmerman, L. F. Soule, M. T. Butt, V. A. Sedlak, C. B. Willingham, and F. D. Rossini, J. Research NBS 41, 323 (1948) RP1929.

[6] A. F. Forziati, B. J. Mair, and F. D. Rossini, J. Research NBS 35, 513 (1945) RP1685.

[7] A. F. Forziati, W. R. Norris, and F. D. Rossini, Bureau of Standards (publication pending).

Washington, June 3, 1949. 